\title{
Blood gas testing and related measurements: National recommendations on behalf of the Croatian Society of Medical Biochemistry and Laboratory Medicine
}

\author{
Lora Dukić*1,2, Lara Milevoj Kopčinović1,2, Adrijana Dorotić1,3, Ivana Baršić1,4 \\ ${ }^{1}$ Croatian Society of Medical Biochemistry and Laboratory Medicine, Committee for the Scientific Professional Development, Working \\ Group for Blood Gas Testing \\ 2University Department of Chemistry, Medical School University Hospital Sestre milosrdnice, Zagreb, Croatia \\ ${ }^{3}$ University Hospital for Infectious Diseases “Dr. Fran Mihaljević”, Department of Medical Biochemistry and Haematology, Zagreb, \\ Croatia \\ ${ }^{4}$ Department of Laboratory Diagnostics, University Hospital Centre Zagreb, Croatia
}

*Corresponding author: lora.dukic@gmail.com

\begin{abstract}
Blood gas analysis (BGA) is exposed to risks of errors caused by improper sampling, transport and storage conditions. The Clinical and Laboratory Standards Institute (CLSI) generated documents with recommendations for avoidance of potential errors caused by sample mishandling. Two main documents related to BGA issued by the CLSI are GP43-A4 (former H11-A4) Procedures for the collection of arterial blood specimens; approved standard - fourth edition, and C46-A2 Blood gas and pH analysis and related measurements; approved guideline - second edition.

Practices related to processing of blood gas samples are not standardized in the Republic of Croatia. Each institution has its own protocol for ordering, collection and analysis of blood gases. Although many laboratories use state of the art analyzers, still many preanalytical procedures remain unchanged.

The objective of the Croatian Society of Medical Biochemistry and Laboratory Medicine (CSMBLM) is to standardize the procedures for BGA based on CLSI recommendations. The Working Group for Blood Gas Testing as part of the Committee for the Scientific Professional Development of the CSMBLM prepared a set of recommended protocols for sampling, transport, storage and processing of blood gas samples based on relevant CLSI documents, relevant literature search and on the results of Croatian survey study on practices and policies in acid-base testing.

Recommendations are intended for laboratory professionals and all healthcare workers involved in blood gas processing.
\end{abstract}

Key words: blood gas analysis; error; guideline; standardization

\section{Introduction}

The term "blood gas analysis" (BGA) is used for laboratory testing that relates to acid-base balance and oxygenation status of a patient. Oxygenation status is assessed using partial pressure of $\mathrm{O}_{2}\left(\mathrm{pO}_{2}\right)$ and haemoglobin oxygen saturation $\left(\mathrm{sO}_{2}\right)$. Partial pressure of $\mathrm{O}_{2}\left(\mathrm{pO}_{2}\right)$ is measured by amperometry, while $\mathrm{sO}_{2}$ is measured by co-oximetry. Blood gas analysers that do not have integrated co-oximeter, report $\mathrm{sO}_{2}$ estimated from $\mathrm{pO}_{2}$ and other parameters. Potentiometry is used for $\mathrm{pH}$ and partial pressure of $\mathrm{CO}_{2}\left(\mathrm{pCO}_{2}\right)$ measurement. Other reported parameters like total $\mathrm{CO}_{2}\left(\mathrm{tCO}_{2}\right)$, bicarbonate con- centration $\left(\mathrm{CHCO}_{3}^{-}\right)$and base excess $(\mathrm{BE})$ are calculated from the measured values. Current analyzers measure not just blood gas parameters but electrolytes (sodium, potassium, chloride, ionized calcium, ionized magnesium) and metabolites (glucose, lactate, bilirubin, creatinine). These additional analytes are usually named as "related measurements" (1). According to the document issued by the Croatian Chamber of Medical Biochemists (CCMB), BGA is categorized as first priority analysis which should be reported within 30 minutes (2). 
Emergency and intensive care departments use BGA as indispensable part of assessment of patient's clinical status (3). Stressful and hectic environment often multiplies probability of errors which could result in the adverse patient outcomes (4). Incentive for preparation of recommendations in this field of laboratory diagnostics was created by the Working Group for Blood Gas Testing (WG BGT) of the Croatian Society of Medical Biochemistry and Laboratory Medicine (CSMBLM). The survey on policies and practices of BGA in Croatia facilitated the identification of crucial issues (5). Survey results showed great heterogeneity in procedures related to BGA in Croatian laboratories. Standardization of ordering, collection and analysis are needed to achieve higher level of laboratory diagnostics of blood gases and related measurements. Based on these crucial points and current global guidelines, members of the WG BGT made joint effort in establishment of recommendations for blood gas testing, covering the preanalytical phase of blood gas testing, sampling procedure, BGA and quality control. Due to the wide use of Point of Care Testing (POCT) instruments for blood gas testing, special attention is required to protocols related to their use by non-laboratory personnel. The POCT Working Group of CSMBLM will prepare the national recommendations for that part of laboratory diagnostics.

The national recommendations for BGT on behalf of the CSMBLM are intended for laboratory professionals and non-laboratory personnel involved in BGT.

This document is divided in the following sections:

1. Sample types used for blood gas analysis,

2. Responsibilities in blood gas testing,

3. Procedure for blood gas sampling, and

4. Blood gas sample analysis.

Specific recommendations of the Working Group are labelled by the boxes. Each recommendation is followed by relevant data from literature.

\section{Sample types used for BGA}

Different sample types can be used for blood gas measurement - arterial blood, "arterialized" capil- lary, "mixed" venous blood and venous blood (1) Clinician should consider the patient's condition, advantages and limitations of various sample types when deciding on the most suitable sample type and collection site (6).

\subsection{Arterial blood}

Arterial blood is the sample type required for correct assessment of gas exchange, function of the lungs $\left(\mathrm{pO}_{2}\right.$ and $\left.\mathrm{pCO}_{2}\right)$, specifically oxygenation status. Composition of the arterial blood is uniform and it is not dependent on changes in systemic or local circulation. Arterial blood is the preferred sample type for evaluation of respiratory status, as well as being suitable for metabolic acid-base disorders.

Sample collection can be performed by using specifically designed arterial blood sample syringes or through arterial line (1). Usual sites of arterial puncture are radial, brachial or femoral artery (Figure 1.) $(6,7)$. Puncture site has to be aseptic (7).

Criteria for the selection of the puncture site are following: presence of adequate collateral blood flow, size and accessibility of the artery (7). (a)

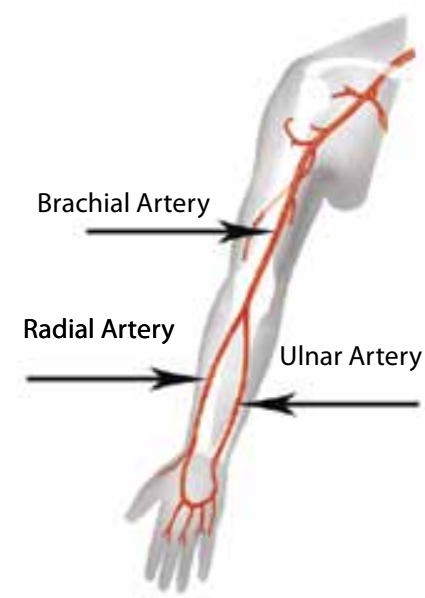

(b)

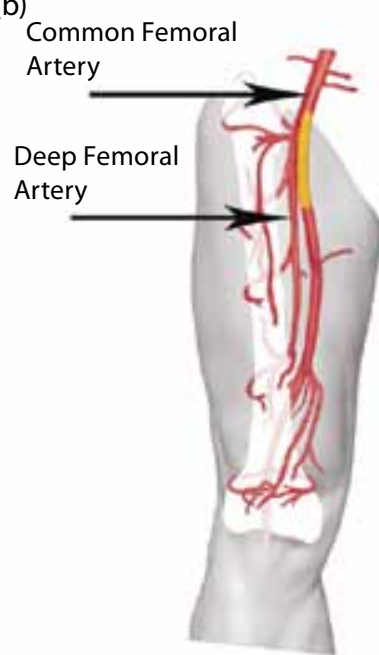

Figure 1. Main arteries.

(a) arm - radial and brachial arteries are puncture sites whereas collateral circulation to the hand is provided by the ulnar artery. (b) groin - femoral artery is the puncture site (figure shows anatomically sub-divided parts of the femoral artery - common and deep femoral artery). 


\subsubsection{Radial artery}

The radial artery is the most commonly used site for arterial puncture. It is easily accessible at the thumb side of the wrist, but because of its small size, use of this artery requires an extensive experience in arterial blood sampling $(7,8)$. Collateral circulation to the hand is normally provided by the ulnar artery, which can be absent in some individuals.

\subsubsection{Brachial artery}

The brachial artery may be preferred for larger volumes of arterial blood. It is more difficult to puncture due to the deeper location between muscles and connective tissues. It is not easily accessible in obese patients, and is not commonly used in children and infants (7).

\subsubsection{Femoral artery}

The femoral artery is a large vessel which is easily palpated and punctured, but it is rarely used in clinical practice due to the poor collateral circulation and increased chance of infection and hematoma (7). The computed tomography (CT) images have revealed a high frequency of small hematoma (47.4\%) around the femoral artery after arterial blood sampling (9).

Collection of arterial blood has its disadvantages. It is more difficult to obtain than venous or capillary blood sample, it is more painful for the patient and it can be potentially dangerous, if the correct procedure is not followed (1). Risks related to arterial blood collection include pain perceived by patient and serious infections due to highly invasive manoeuvre. Moreover, a haemostasis not perfectly performed could be a source of damage, hematomas and haemorrhagia. Usual complications of the procedure are the following: arteriospasm, hematoma, nerve damage, fainting and drop in blood pressure (10).

\section{2 “Arterialized" capillary sample}

A capillary blood sample can be considered as an adequate substitute for the arterial blood in neonatal and paediatric units. A capillary blood sample can replace an arterial sample only when an arterialisation technique is used, although entirely reliable $\mathrm{pO}_{2}$ measurement can be achieved only when an arterial blood sample is used. Skin puncture is also applicable for adult patients with severe burns, tendencies to thrombosis, obese and geriatric patients.

According to the survey study on practices and policies in acid-base testing in Croatian laboratories, a capillary sample is a sample type used in most of the institutions in Croatia. Survey results showed that 19 out of 41 participants (or 0.46) have a capillary sample taken for BGA in an adult patient population. The same survey showed that only 7 laboratories out of 33 (or 0.21 ) analyze exclusively arterial samples for BGA. Avoidance of arterial sample collection can be attributed to economic factors and difficulty of collection. Many physicians are accustomed to order capillary BGA as a part of the regular patient follow-up together with biochemistry and haematology testing (5).

Capillary blood collection is a simple, relatively painless procedure that can be performed after a minimal training of medical personnel, but it has its disadvantages. Zavorsky et al. compared laboratory results of BGA conducted in arterial and capillary blood samples. Results showed that arterialized capillary blood is a suitable alternative for measurement of $\mathrm{pH}$ and $\mathrm{pCO}_{2}$ over a wide range of values, but it is not suitable for $\mathrm{pO}_{2}$ (11). Arterial blood $\mathrm{pO}_{2}$ is physiologically higher than the arterialized capillary $\mathrm{pO}_{2}$ because of the oxygen consumption in skin capillary bed. The possibility of arterialized capillary sample accurately predicting arterial $\mathrm{pO}_{2}$ is improved when the arterial $\mathrm{pO}_{2}$ is low because arteriovenous difference is reduced in hypoxia (11). There are some circumstances in which arterial blood cannot be collected directly and capillary blood sample can be considered as an adequate substitute for the arterial blood $(1,8,12)$. In neonatal and paediatric units capillary blood sample is desirable sample type because infants and small children have small total volume of blood and frequent blood collection can result in anaemia $(8,12-$ 14). Skin puncture is also applicable for adult patients with severe burns, tendencies to thrombosis, obese and geriatric patients $(8,12)$. 
A capillary blood sample can replace arterial sample (but not for $\mathrm{pO}_{2}$ ) only when an arterialisation technique is used (1). A warmed capillary sample approximates an arterial specimen $(1,7)$ because portion of arterial blood in capillary sample can be increased up to seven fold by using an arterialisation technique (12). This technique consists of use of a warm, moist towel at a temperature not higher than $42{ }^{\circ} \mathrm{C}$ on a puncture site not longer than three to five minutes (12) or use of other vasodilator agent (6).

\subsubsection{Puncture site}

Recommended puncture site for capillary sampling is the fingertip or heel, depending on the patient age (12). Lateral or medial plantar surface of the heel is the puncture site for neonatal population, while the fingertip is recommended for paediatric population $(1,12)$.

Recent studies show earlobe sampling has advantage over fingertip capillary sampling when capillary blood gas sampling for an adult patient is considered.

The CLSI document on a capillary sample GP42-A6 recommends capillary sampling be performed by puncturing the skin on a finger or heel, depending on the age of the patient. In neonates, the recommendation is to perform punctures on the lateral or medial plantar surface of the heel. In paediatric population the recommended site for capillary sample collection is the fingertip $(1,12)$. Earlobe as a puncture site is used in mass screening or research studies and for adult patients. The World Health Organization and CLSI guidelines on capillary blood sampling state that earlobes are unsuitable sites for blood sampling in neonatal and paediatric population $(8,12)$. Some authors consider the results from earlobe sampling more reliable than results obtained by fingertip sampling. Nevertheless, arterialized earlobe blood $\mathrm{pO}_{2}$ is not as reliable as arterial $\mathrm{pO}_{2}(11,15,16)$.

\subsubsection{Precaution measures for capillary blood gas sampling}

There are multiple risks for preanalytical errors when collecting capillary blood: gas exchange with air (1); strong, repetitive pressure on puncture site - "milking" should be avoided, as it can cause haemolysis and contamination of the sample with tissue fluid, ultimately resulting in elevated potassium levels and dilution of blood, causing lower values for other electrolytes $(1,17)$. Finally thorough mixing of the blood is necessary to prevent clotting (12).

It is important to be aware of these limitations of a capillary sample when selecting a blood gas collection site.

\subsection{Venous blood sample}

Venous blood is not a suitable substitute for arterial blood in BGA because of the nature of the sample and difference in oxygenation levels compared with arterial blood. It is suitable for the assessment of different variants of haemoglobin, electrolytes, as well as $\mathrm{pH}, \mathrm{pCO}_{2}, \mathrm{HCO}_{3}$ and metabolites (1).

\section{4 “Mixed" venous blood}

"Mixed" venous blood sample is obtained from the pulmonary artery by means of a pulmonary artery catheter and it is used to evaluate oxygen uptake and cardiac output (1).

\section{Responsibilities in blood gas testing}

Arterial blood sampling should only be performed by healthcare workers within the described legal scope of practice for their position in their country and who have demonstrated proficiency after formal training (8).

Based on the Training and skill catalogue for medical study, medical doctors (MD) in Croatia must be able to independently perform an arterial blood sampling after graduation. This skill is further improved during postgraduate studies and speciali- 
zation (18). Bachelor of nursing is trained in sampling of arterial blood ordered by the MD (19). A nurse has theoretical and practical knowledge in sampling other samples that are used in analysis of acid-base status - capillary and venous blood samples (20).

According to the document published by the CCMB in 2013 "Competences for issuing the license for masters of science in medical biochemistry and specialists in medical biochemistry and laboratory medicine", master of science in medical biochemistry post completion of the five year course, one year internship and passing the state license examination, has the theoretical and practical knowledge of capillary blood sampling, performing BGA and interpretation of acid-base balance results. Specialists in medical biochemistry and laboratory medicine have additional qualifications related to implementation of new techniques for sampling and analysis of blood gases, establishment of quality control programs and should have performed at least 1000 analysis of acid-base status (21). They play a key role in education of personnel responsible for sampling which is also regulated by law within Regulations on medical biochemistry practice by the patient's bedside (22).
Table 1 shows the list of healthcare workers in Croatia authorized to carry out specific procedures related to BGA.

Based on previous statements, we can conclude that sampling and analysis of blood gas samples are complex interdisciplinary processes that include a broad spectrum of medical experts, as it was observed from the survey conducted during 2013 by the WG BGT (5).

\section{Procedure for blood gas sampling}

\subsection{Preparation for sampling}

Preparation for sampling includes patient identification, a pathology request form for BGA, patient assessment, explanation of the procedure of sample collection to the patient and regulations related to blood gas sample labelling.

\subsubsection{Patient identification}

\subsubsection{Procedure for the identification of conscious patient}

Personnel responsible for the sampling procedure should ask the patient to state his/her first

TABLE 1. List of healthcare workers in Croatia authorized to carry out specific procedures related to BGT based on the available legislation and practices observed in the Croatian laboratories (5,18-22).

\begin{tabular}{|c|c|}
\hline Procedure & Healthcare worker \\
\hline Arterial blood sampling & $\begin{array}{l}\text { Doctor of medicine } \\
\text { Bachelor of nursing }\end{array}$ \\
\hline Capillary blood sampling & $\begin{array}{l}\text { Bachelor of nursing } \\
\text { Master of science in medical biochemistry } \\
\text { Laboratory technician }\end{array}$ \\
\hline Blood gas sample analysis & $\begin{array}{l}\text { Master of science in medical biochemistry } \\
\text { Laboratory technician } \\
\text { Bachelor of nursing (after education is performed by laboratory personnel) }\end{array}$ \\
\hline Quality control & $\begin{array}{l}\text { Master of science in medical biochemistry } \\
\text { Laboratory technician } \\
\text { Bachelor of nursing (after education is performed by laboratory personnel) }\end{array}$ \\
\hline Result interpretation & $\begin{array}{l}\text { Master of science in medical biochemistry } \\
\text { Doctor of medicine }\end{array}$ \\
\hline Implementation of blood gas equipment & Specialist in medical biochemistry and laboratory medicine \\
\hline Personnel education & Specialist in medical biochemistry and laboratory medicine \\
\hline
\end{tabular}


and last name and date of birth, and then compare the details with the details written on the test request form. Discrepancies should be resolved prior the sampling procedure with the responsible person in the area. Use of at least two identifiers is needed for patient safety.

Positive patient identification is required as stated by the procedure recommended by the Working Group for Pre-analytics of CSMBLM (23), needing at least two identifiers (e.g. full name (first and last name) and date of birth) for patient safety (24).

\subsubsection{Procedure of identification in patient who is unable to give personal details}

If it is not possible to obtain personal details directly from the patient, the person responsible for the patient (caregiver, family member, nurse) should be asked to identify the patient and provide the required details. Name of the verifier should also be documented.

\subsubsection{Procedure for the identification of unidentified emergency patient.}

Master (temporary) identification patient number for the unidentified emergency patient should be defined according to the institutional policy. When a permanent identification number is assigned to the patient, the temporary identification number should be cross-referenced to the permanent number (25).

\subsubsection{Test request form for $B G A$}

The following items should be stated in the test request form: the patient's first and last name, gender, date of birth, personal identification number or health insurance number, date and time and sampling location (hospital ward) $(23,26)$. The test request form should contain the name of the responsible person who requested BGA (27). Contact information of the responsible person is needed for critical result reporting and consulting in the case of suspected non-conformities.
In addition to the abovementioned items, the following information are needed for correct interpretation of BGA:

- collection site and sampling technique (e.g. arterial blood collected by arterial puncture or from arterial catheter),

- patient ventilatory status,

- mode of ventilation and information on oxygen supply,

- respiratory rate, body temperature, patient position and activity, including special remarks (e.g. anxiety),

- name of the person collecting the sample,

- request for related measurements (electrolytes, metabolites) and

- working diagnosis.

\subsubsection{Patient assessment}

For correct assessment of the patient's condition, body temperature (6), respiratory rate and activity including special remarks (e.g. anxiety) need to be recorded (28).

Changes in body temperature have impact on blood gas values because of the shift in chemical equilibrium, while physical activity and anxiety can cause an increase in the respiratory rate $(1,29)$.

\subsubsection{Assessment of ventilatory changes}

It is recommended that patient remains in a relaxed state for at least five minutes before sampling in order to establish stabile state (7).

Urgent blood gas sampling and analysis in critical care settings is performed immediately for prompt evaluation of the patient vital functions.

In situations when ventilatory changes have been made to patients, it is recommended to wait for 20 to 30 minutes to reach the "steadystate" and to obtain reliable blood gas results.

\subsubsection{Explanation of the sampling procedure to the patient}

Patient should be informed in detail about the procedure of specimen collection (30). 


\subsubsection{Sample labelling}

A sample for BGA should be labelled with the patient's full name and second identifier. Institutional policy defines the second identifier, which could be: date of birth, health insurance number or identification number.

A capillary sample should be directly labelled with the label containing two patient identifiers immediately after the sampling procedure and before leaving the patient (31). Figure 2. shows recommended capillary sample barcode labelling. Arterial sample should be directly labelled with the label containing two patient identifiers (32).

\subsection{Arterial blood sampling procedure}

Laboratory personnel are responsible for appropriate written instructions for sample collection according to the ISO 15189 standard (27). Arterial blood collection is performed by physicians (18) and nursing graduates (19) in healthcare institutions in Croatia. In order to ensure arterial sample quality and result reliability, laboratory personnel should inform ward personnel responsible for arterial blood collection of the required procedures.

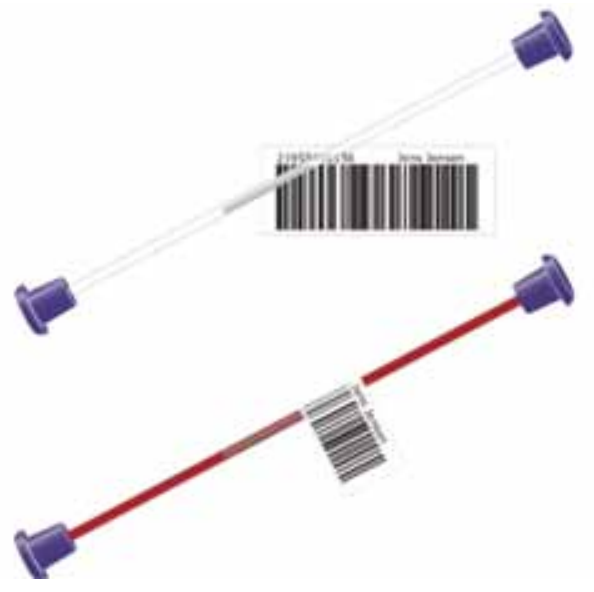

Figure 2. Recommended capillary blood gas sample labelling (source: Wennecke G, Dal Knudby M. Avoiding preanalytical errors - in capillary blood testing. Radiometer Medical Aps, Brønshøj, Denmark, 2009.)
Information and education of clinical and nursing personnel encompasses the following issues:

- Sample device,

- Anticoagulant,

- Sampling procedure - arterial puncture and collection of a sample from arterial catheter (air bubbles expulsion; sample mixing; flushing of the catheter),

- Sample labelling and test order form,

- Transport and

- Storage.

\subsubsection{Sample device}

Arterial blood sampling device, as stated in CLSI C46-A2 document, should be a plastic, disposable, self-filling syringe. Self-filling syringes fill spontaneously with arterial punctures. Collector needs to be aware of the minimal volume needed for reliable measurement of all requested parameters $(32,33)$. Minimal blood volume in the syringe is recommended to ensure accurate results are obtained and to cause minimal blood loss for the patient.

\subsubsection{Anticoagulant}

Use of lyophilized, balanced lithium heparin is recommended for BGA and related measurements in arterial and capillary blood samples. It is acceptable to use anticoagulant in spray and dried form. Sodium heparin is not recommended as it causes falsely high sodium values. Use of liquid (therapeutic) heparin should be avoided because of dilution effect on blood gas and related parameters and ability to bind electrolytes, namely calcium. Erroneous results can arise from improper anticoagulant concentration and insufficient mixing of anticoagulant and blood sample (33-35). Different preparations of heparin by syringe manufacturers can be a source of variability of BGA results (36).

\subsubsection{Sampling procedure - arterial blood gas sample}

Examination of collateral circulation is obligatory when arterial blood sampling is performed from 
the radial artery. After aseptic preparation of puncture site, dedicated collection device designed for arterial collection should be used. This procedure is needed to avoid accidental contamination of arterial sample with venous blood.

When the syringe has been filled with adequate blood, withdraw the syringe, place a dry gauze sponge on the puncture site and press firmly on the puncture site for 3-5 minutes. Arterial blood should be collected under anaerobic conditions to minimize air exposure (1).

Air bubbles should be immediately expelled from the syringe by gently tapping the sides of the syringe allowing them to go to the top of the syringe for expulsion in order to avoid an air contamination and falsely altering the $\mathrm{pO}_{2}$ results (37).

Following air-bubbles expulsion, homogenization of the sample with the anticoagulant should be done to avoid formation of the clots. For optimal anticoagulation, rolling of the syringe between the palms, and then inverting it vertically is recommended (Figure 3.).

Warning: Vigorous mixing can cause significant changes in blood gas and related measurement values due to haemolysis $(38,39)$.

\subsubsection{Sampling from arterial catheter}

During sampling from an arterial catheter, there is a risk of contamination of specimen with intravenous solution, medication or diluting the sample with electrolyte solution (e.g. $\mathrm{NaCl}$ ) that may be in

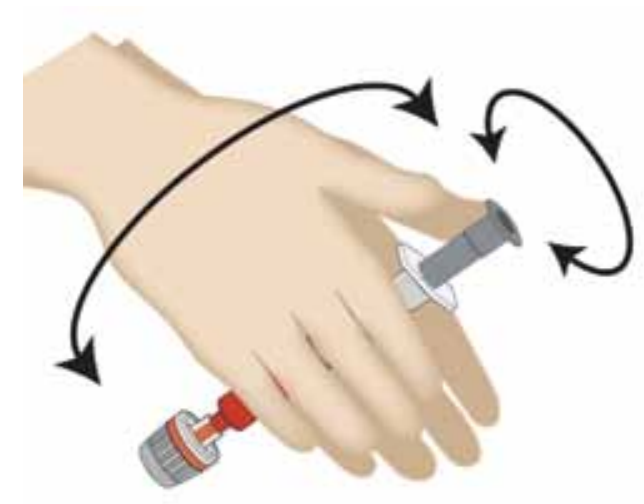

Figure 3. Mixing of the arterial blood sample (source: Wennecke G, Juel G. Avoiding preanalytical errors - in blood gas testing. Radiometer Medical Aps, Brønshøj, Denmark, 2008.) the catheter (1). If the catheter is not flushed sufficiently, sodium and chloride will be elevated whereas all other analytes will be falsely lower (32).

When an arterial specimen is to be collected from an arterial catheter, the catheter must be flushed prior the sampling. Recommended flushing volume is at least 3 times the dead volume of the catheter. Manufacturer's specification for the required dead volume is recommended to be used for the catheter.

\subsubsection{Transport}

Samples for BGA should be analyzed as soon as possible. Arterial samples taken into standard, recommended plastic syringes should be analyzed within 30 minutes. Hand transport of samples without sudden, vigorous movements is recommended. Risk of haemolysed and air-contaminated sample is higher if the sample is subjected to shaking. Transport of samples on ice is not recommended because plastic syringes are more permeable to gases at lower temperatures and due to the high risk of haemolysis. Exact times of the sampling and delivery of the sample to central laboratory for analysis should be recorded. If the time between sampling and delivery to the laboratory exceeds the allowed stability interval (30 minutes), non-conformity should be recorded and ward personnel informed on need to recollect.

\subsubsection{Storage - arterial blood gas samples}

Due to cellular metabolism in whole blood specimen, blood gas and related parameters can change rapidly and significantly, so immediate analysis upon collection is highly recommended. Samples with high leukocyte and platelet count and samples intended for special studies (e.g. shunt analysis) need to be analyzed within 5 minutes.

BGA within 30 minutes gives reliable results except in the presence of an elevated leukocyte or platelet count (40). In that case, glass syringe 
should be used (if available) and immersed into mixture of ice and water (41) or recollection and immediate transport for analysis.

\subsection{Capillary blood sampling procedure}

Sample collection should be as described in the national recommendations of the CSMBLM for capillary blood sampling (42).

Capillary sampling intended for BGA is recommended for neonatal and paediatric population in the first instance. Capillary blood gas sampling is recommended for adult patients only in situations when arterial blood puncture cannot be performed (patients with severe burns, tendencies to thrombosis, obese and geriatric patients).

General recommendations regarding the location of skin puncture are: (a) the puncture must be performed on the palm-up surface of the distal segment (fingertip) of the middle or ring finger, (b) the middle finger and ring finger are recommended for capillary sampling, while the thumb and index finger are more sensitive and calloused, (c) the skin of the smallest finger is very thin, so this finger is not recommended for capillary sampling.

It is not allowed capillary sampling from fingers in neonatal population, but from the side of the heel.

\subsubsection{Earlobe skin puncture}

Despite the fact that current CLSI and WHO guidelines do not give priority to earlobe skin puncture, objective evidence point out the advantages of this puncture site in adult patients compared to the fingertip in cases when arterial blood sampling is not feasible. Blood adequately sampled from an earlobe resembles more closely the composition of arterial blood in comparison to blood sampled from a fingertip $(11,15,16)$. When the earlobe puncture site is selected, capillary blood sampling procedure needs to be performed according to the national recommendations for capillary blood sampling (42).

\subsubsection{Arterialisation of the skin puncture site for capillary sampling}

It is highly recommended that the puncture site be arterialized prior to capillary sampling (42). This procedure will give similar results to the ideal sample, arterial blood gas sample.

To achieve arterialisation, the puncture site should be covered with a warm, moist towel heated at 42 ${ }^{\circ} \mathrm{C}$ or less for 3-5 min prior to puncture. This is the recommended arterialisation method because its application does not produce adverse effects.

Vasodilatory creams based on capsaicin, or nicotinate and salicylate or $2 \%$ nitroglycerine cream can be used, but precaution measures are needed due to potential adverse effects listed in package insert. Application of vasodilatory cream is performed by spreading of cream on the puncture site prior sampling. Following application, cream should be left on puncture site until becomes red and warm, which can take from 5 to 20 minutes. After proper arterialisation is achieved, the puncture site should be cleaned with the clean gauze and capillary blood gas sampling can be performed. All the steps in capillary sampling are described in the national recommendations by CSMBLM for capillary sampling (42). These steps include:

- Disinfection of the puncture site with $70 \%$ aqueous solution of isopropanol;

- Air drying of the puncture site;

- Skin puncture and wiping away of the first drop and

- Anaerobic collection of the blood from the centre of the second spontaneously formed blood drop.

Applying pressure ("milking") of the puncture site during the sampling should be avoided. Consequences of "milking" are haemolysis and sample contamination with tissue fluid.

Each laboratory should follow minimal volume specifications defined by the blood gas analyzer manufacturer and use appropriate capillary tubes. Balanced lyophilized lithium heparin is the recommended anticoagulant. Spray and dried form of this anticoagulant are acceptable. 
Anaerobic filling of blood sample is needed in order to prevent changes in results caused by atmospheric air contamination of the sample. When capillary filling is completed, immediate capping should be performed in order to avoid exposure of the sample to air. Thorough mixing with the anticoagulant is needed in order to prevent clotting. For that purpose, metal mixing bar and magnet are used. National recommendations on behalf of CSMBLM for capillary blood sampling advises the capillary end that is in direct contact with the blood to be capped first, insert a metal mixing bar, and then cap the other end of capillary. A magnet should be moved from one end of the capillary to the other for five times. In this way sample is homogenised with the metal mixing bar while preventing clot formation (42).

\subsubsection{Capillary blood sample transport and storage}

The general recommendation is to analyze the capillary sample immediately. Samples collected in capillary tubes should be analyzed within 10 minutes. Samples should be kept at room temperature until analysis. If storage is longer, samples should be collected in glass capillary tubes and stored horizontally at $0-4{ }^{\circ} \mathrm{C}$ (immersed into mixture of ice and water to avoid haemolysis) for a maximum of 30 minutes.

\section{Blood gas sample analysis}

\subsection{Personnel education and training}

BGA should be performed by trained laboratory personnel. Complete training requirements should be documented in laboratory standard operating procedures. The competence of employees responsible for BGA should be evaluated for new employees and the competence reevaluated annually. Training and competency records for all staff should be retained as per requirements of good laboratory practice.

\subsection{Confirmation of patient's identification}

Time of the delivery of the blood gas sample to the central laboratory should be recorded. Time between sampling and analysis should not exceed 30 minutes. If it exceeds the recommended time interval, clinical staff should be informed and recollection requested.

Prior to analysis, the employee responsible for sample analysis should check the detail on the specimen label match those on the test request form to confirm the patient's identification. If the specimen needs to be immersed into an ice slurry (mixture of ice and water) until the analysis can be performed (i.e. if delay of more than 30 minutes is anticipated), the label integrity must be protected even during immersion (7).

\subsection{Sample preparation prior to analysis}

The quality of the whole blood sample should be inspected before analysis. Blood gas samples containing air bubbles or visible clots are unacceptable for analysis.

Proper mixing of whole blood samples is critical for obtaining accurate haemoglobin results.

Capillary samples should be mixed using a metal bar and magnet. The magnet should be moved from end to end over the capillary until the blood components are uniformly distributed (homogenized) or for at least 5 seconds. One end of the capillary should be opened by carefully removing the sealant cap. The metal bar should be removed by pulling the magnet slowly over the capillary, being careful not to spill blood or introduce air into the sample. Before introducing the sample into the analyzer, the opposite end of the capillary should be opened by removing the remaining sealant cap and the sample should be allowed to flow to the end to remove trapped air. The capillary should then be presented to the instrument nozzle, adapter or aspirator tip. Analysis should be initiated as described in the specific instrument instructions. 
According to CLSI (1) recommendations arterial samples should be gently rotated, either manually or using a mechanical device that rotates the sample through two axes, for a minimum of one minute immediately prior to analysis.

Specimen integrity checks should be performed before analysis: in case of air bubbles presence, clots, insufficient sample volume or any other nonconformities, analysis should not proceed and recollection should be requested.

Blood used for the measurement of blood gases and related analytes should be thoroughly mixed immediately prior to analysis to achieve a homogeneous sample prior to insertion of sample into the instrument. The process of mixing should not alter the integrity of the sample, causing haemolysis, and thus affect the quality of results $(1,34)$.

Shorter mixing intervals are acceptable if only one or two minutes (or less) have elapsed since the arterial sample collection. Stored syringe samples should be mixed by inverting the syringe 10 times and then rolling it horizontally between the palms for 10 seconds. Upon storage, samples tend to settle and may require a longer mixing time to insure homogeneity (43).

Incomplete dissolution of anticoagulant in the syringe due to inadequate mixing immediately after collection can result in the formation of blood clots. The presence of clots in the sample renders it non-homogeneous and can affect the accuracy of analysis. Furthermore, clots can block the pathway of the blood gas analyzer and give either confounding results or render the analyzer inoperable. Potassium concentrations measured by blood gas analyzer can be spuriously increased due to haemolysis during clot formation.

\subsection{Sample analysis}

To ensure patient safety, patient data should be entered in the analyzer or laboratory information system (manually or using the bar-code reader, if available) and checked according to the manufacturers' instructions. Time of the sample's analysis should be recorded.
Data on patient's body temperature and oxygen supply can be entered in some analyzers, enabling the correction of obtained BGA results to patient's current body temperature and for special calculations for which data on oxygen supply are needed. These additional features should be implemented only after adequate education of personnel involved in BGA.

The $\mathrm{pH}$ of freshly drawn blood decreases on standing at a rate of 0.02 to $0.03 \mathrm{pH}$ unit/h at $22^{\circ} \mathrm{C}$ and $<$ $0.01 \mathrm{pH}$ unit/hour at $4{ }^{\circ} \mathrm{C}$. This decrease in $\mathrm{pH}$ is accompanied with a corresponding decrease in glucose and increase in lactate and $\mathrm{pCO}_{2}$ in the sample mainly due to glycolysis and cell respiration. Unwanted effects on $\mathrm{pH}$ and blood gases can be prevented by analyzing the sample immediately, within 30 minutes from collection.

Samples with elevated white blood cell counts must be analyzed immediately due to the marked effect of increased glycolysis in $\mathrm{pH}$, blood gases, and lactate $(44,7)$.

When syringe samples are introduced into the analyzer by aspiration, an air bubble will form in the remainder of the sample. Expel the air immediately in case the measurement needs to be repeated.

The sample should be reanalyzed immediately (preferably on another analyzer if available) if questionable results are obtained (i.e. inconsistent with the patient's past results or current clinical status), before the quality of the sample deteriorates rapidly (1).

To ensure traceability after analysis, BGA samples should be stored as described by the CCMB document "Sample storage and preparation" (45).

\subsection{Instrumentation' maintenance}

The wide availability of instruments and calibration materials has made reliable and accurate measurement of the available analytes mainly dependent on regular maintenance, adherence to manufacturer's recommended procedures, control of the equipment and proper collection and handling of samples. Each laboratory should follow the manufacturer's recommendations for maintenance for optimal analyzer performance. 


\subsection{Results reporting}

In addition to blood gas (and/or other analytes) values and patient's identification data, a complete report should contain information about:

- collection time,

- delivery time,

- analysis time,

- results release time,

- sample type (i.e. arterial, capillary) and sampling mode,

- any ventilator settings, mode of ventilation and information on oxygen supply,

- the type and location of any fluid infusions,

- the collection site,

- respiratory rate,

- body temperature and

- the patient's posture and activity with special remarks on patient status (e.g. anxiety).

Comments regarding quality of the sample, transportation delays and improper storage should also be documented.

\subsection{Analytical interferences}

Laboratories need to be familiar with the analyzer limitations, especially those regarding potential interferences.

Polymeric $\mathrm{pH}$ sensors, typically used in cartridgebased analyzers, as well as blood gas and related analytes sensors, are subject to interferences from lipophilic compounds like perfluorocarbon found in emulsion based quality control (QC) materials.

Cationic surfactants, especially benzalkonium compounds, may interfere with sodium, potassium and ionized calcium measurements (46). Ionized magnesium sensors are subject to ionized calcium and sodium interference. Thiocyanate concentrations commonly present in smokers may cause a decrease in ionized magnesium concentrations (1). Chloride sensors are based on ion exchange membranes and thus are susceptible to interferences caused by more lipophilic anions (other than chloride) present in the sample. Salicylates, thiocyanates, bromides and iodides produce false- ly elevated chloride concentrations. If chloride electrode is repeatedly exposed to the anticoagulant heparin (e.g. when attempting to wash out clots from the analyzer), due to its negative charge it could be extracted to the chloride membrane resulting in the reduction of membrane sensitivity for chloride ions.

Sensors for glucose and lactate are susceptible to interference from endogenous and exogenous oxidizable substances present in blood (uric acid, ascorbic acid, acetaminophen, and dopamine). Fluoride and oxalate, typically used as additives in blood collection tubes, could produce a negative interference on glucose and lactate biosensors by inhibiting the activities of oxidase enzymes used in the measurement reaction.

Variation in electrolyte and protein concentrations may interfere with haematocrit conductivity measurements.

The presence of fetal and rarely sulfhaemoglobin (i.e. aberrant haemoglobins) produces interferences with co-oximetry measurements. The therapeutic administration of hydroxycobalamin and methylene blue (which are coloured and interfere with spectrophotometric measurements used in co-oximetry) may affect haemoglobin fraction measurement (1).

Haemolysis has a significant effect on potassium concentrations because of the concentration gradient present between cells (erythrocytes, leucocytes and platelets) and plasma. Haemolysis may cause erroneously low sodium and ionized calcium concentrations due to dilution effect. Other analytes may be affected as well, depending on the level of haemolysis $(1,38)$. Although the presence of haemolysis in whole blood samples is impossible to detect visually, it has been shown to cause unreliable $\mathrm{pO}_{2}$ and $\mathrm{pCO}_{2}$ results (i.e. significantly lower $\mathrm{pO}_{2}$ and higher $\mathrm{pCO}_{2}$, respectively). Furthermore, haemolysis may cause a clinically important decrease in $\mathrm{sO}_{2}$ and carboxyhaemoglobin $(\mathrm{COHb})$. If a result is obtained that does not reflect the patient's condition, the presence of haemolysis should be suspected and investigated by transferring the whole blood sample into an additive free tube and expose it to rapid centrifugation 
$(28,38)$. Sample lipemia interferes with the measurement of total haemoglobin and haemoglobin fractions $(1,34)$.

Methods for the measurement of haematocrit on blood gas analyzers include calculation from the measured total haemoglobin concentration or its measurement by conductivity. The conductivity measurement is dependent on electrolyte concentrations and thus susceptible to its variations. Additionally, abnormal protein concentrations (i.e. low protein concentrations resulting from blood dilution with electrolyte solutions) result in spuriously low haematocrit values measured by conductivity. The calculation of haematocrit from measured total haemoglobin concentration assumes a normal MCHC value (about $340 \mathrm{~g} / \mathrm{L}$ ). Disorders resulting in abnormal MCHC values (microcytic and/or macrocytic anaemias, hereditary spherocytosis, and sickle cell disease) influence the calculation's accuracy. MCHC values can be affected by erythrocytes aggregation due to presence of agglutinins.

The absorption spectra of fetal and adult haemoglobin differ slightly and the presence of elevated concentrations of fetal haemoglobin may cause falsely elevated carboxy- and/or methaemoglobin concentrations. Some analyzers can correct for the presence of fetal haemoglobin $(1,28)$.

The choice of anticoagulant can affect blood gas and related measurements. It has been already stated that the anticoagulant of choice in blood gas and related measurements is lyophilized, balanced heparin. Spray and dried form of anticoagulant is acceptable, too. If liquid heparin is used instead, it may cause erroneous results by dilution effect on all analytes and/or interfere with ionized calcium measurements by binding it. Sodium heparin may falsely increase sodium concentrations. The use of therapeutic heparin for BGA is not recommended since it may alter ionized calcium, magnesium and $\mathrm{pH}$ measurements. EDTA and oxalate anticoagulants are not used for blood gas analysis because they may interfere with electrolyte or enzymatic measurements by chelating cations $(1,28)$.

Lubricants and additives could be present in syringes that may interfere with co-oximetry meas- urements. Syringe and instrument manufacturers should be contacted for further specific information (1).

Contamination of the sample with "line draw" fluid from an indwelling catheter may lead to clinically significant dilution errors (28).

\subsection{Calibration}

Operators should adhere to analyzer specific calibration procedures recommended by the manufacturer. Calibration materials should be traceable to certified reference materials. To meet acceptable analytical performance for clinical application, traceable QC materials and a proper proficiency testing program should be instituted (1).

Each analyzer has recommended calibration protocols that include specific calibration materials and frequency (depending on the stability of the technology used for measurement). Many analytical systems have a built-in calibration check which is performed after every system calibration. This process allows timely error detection before running QC and patient samples. Unfortunately, due to matrix effects, the recovery of expected calibrator values may not accurately reflect the performance with patient samples (1).

The traceability of calibrators used should be documented by a manufacturer's statement regarding traceability and associated uncertainties. Primary $\mathrm{pH}$ standards should be traceable to the reference method for $\mathrm{pH}$, calibrated with Standard Reference Material (SRM) 186 I and 186 II. Manufacturers should use blood tonometry with gas mixtures of certified composition (SRM available from National Institute of Standards and Technology, NIST) as a reference method for establishing accuracy for $\mathrm{pO}_{2}$ and $\mathrm{pCO}_{2}$ measuring devices. Haemoglobin fractions are based on absorbance ratios and do not require separate calibrations if the measuring wavelengths remain unchanged. Primary standards for potassium, sodium and chloride determination are gravimetric solutions produced from $\mathrm{KCl}$ and $\mathrm{NaCl}$ salts of high purity traceable to SRM 918, SRM 919 and SRM 999, respec- 
tively. The primary ionized calcium standard is produced from $\mathrm{CaCO}_{3}$ buffered to $\mathrm{pH}=7.4$ and traceable to NIST SRM 915. The primary glucose standards are traceable to SRM 917a (D-glucose). Their glucose concentration is assigned using the glucose reference method. Since no certified SRM for lactate is available, primary standards are prepared from a pure commercially available lithium lactic salt.

If traceable standards do not exist for a particular analyte, traceability requirements may be met by using certified materials of known purity with reliable physical and chemical characterization, use of specified methods and/or consensus standards or participation in a suitable program of inter-laboratory comparisons (1).

\subsection{Quality control for blood gas analysis and related measurements}

Quality control (QC) for blood gas and related measurements is particularly important because these tests are requested predominantly in critically ill patients and treatment is instituted immediately upon test results receipt. Internal QC verifies the attainment of analyzer's analytical performance to the appropriate criteria for intended use $(27,47)$.

Each laboratory should design the internal QC analysis strategy in accordance with requirements of good laboratory practice.

It is recommended to perform the simultaneous analysis of at least two levels of commercial QC materials daily to evaluate the analytical quality of the measurement system. QC materials should cover the measuring range and possibly the critical clinical points. QC materials independent of those produced by the analyzer' manufacturer is suggested but this may not be attainable with cartridge based analysers.

Procedures to be followed in case of unacceptable QC results should be documented and instituted as required.

If multiple blood gas analyzers are used for BGA and related measurements, comparisons using patient's samples should be implemented as a useful adjunct to daily QC testing. At least one daily comparison of each analyte measured is recommended.

The recommendations stated above represent the minimal QC requirements for blood gas and related measurements. Laboratories are strongly encouraged to define their own quality goals for BGA and related measurements and then tailor specific QC protocols to ensure that every patient's test result meets the stated quality goal $(27,47)$. QC results must be reviewed and acted upon in a timely manner, as per laboratory procedures, to assure confidence of reported results. The critical question in blood gas QC protocols is the strategy of QC testing (i.e. number, type and frequency). QC frequency is highly dependent on frequency of BGT (i.e. workload), acceptability of quality control results, manufacturer's recommendations and regulatory requirements. For example, the CLIA requirements for BGA are stringent: at least one QC sample every 8 hours of testing and 3 levels (low, normal and high) each 24 hours of testing. The practical answer would be to run on every analyzer in use, at least once per 8 hours, 3 levels of control for $\mathrm{pH}, \mathrm{pO}_{2}$ and $\mathrm{pCO}_{2}$, and additionally, always on completion of maintenance and troubleshooting procedures $(27,44,47,48)$.

If multiple blood gas analyzers are closely located in the laboratory or hospital site, a single sample should be analyzed on a number of analyzers, within a short period of time, considering sample lability. If analysers, which measure $\mathrm{sO}_{2}$ by co-oxymetry, and those which calculate $\mathrm{sO}_{2}$ are used interchangeably in the same laboratory, the comparability of measured and calculated $\mathrm{sO}_{2}$ results should be verified. If multiple blood gas analyzers are dislocated in a laboratory or hospital site, patient samples should not be used. Instead, the acceptable analytical performance of the analyzer should be verified using established internal QC protocols.

If blood gas analyzers are located closely to the main chemistry analyzer, and measurements of electrolytes and/or metabolites from serum/plasma and whole blood samples are performed in 
the laboratory, an ongoing comparison of the two platforms should be instituted. It should be performed by analyzing serum/plasma samples on multiple measurement systems. The differences in measuring techniques and sample types should be taken into consideration when interpreting the obtained bias (44).

Commercially available QC materials for BGA and related measurements include blood-based solutions containing free haemoglobin, emulsionbased and buffered aqueous fluid control materials. They may be introduced by the operator or integrated in the analytical system and analyzed automatically (1).

Buffered aqueous QC solutions typically simulate blood with respect to $\mathrm{pH}$ and $\mathrm{pCO}_{2}$, but have low oxygen buffering capacity and therefore poorly resist $\mathrm{pO}_{2}$ changes, which makes them susceptible to storage and handling temperature variability. Although most commonly used, the disadvantage of aqueous QC materials originates from their dissimilarity with blood (non-commutability) in terms of viscosity, surface tension and electrical conductivity $(1,44)$. Haemoglobin-containing QC materials consist of red blood cells or haemolysate, which enhances the $\mathrm{pO}_{2}$ buffering. Emulsion controls contain oils (typically perfluorocarbon) in buffered aqueous salt solutions. While they are better in resisting oxygen change than aqueous solutions, their surface tension and density are not identical to that of blood. QC materials sealed in ampoules contain a gas mixture phase in the headspace and must be equilibrated to the appropriate temperature before opening (if room temperature is significantly different from $25{ }^{\circ} \mathrm{C}$ ). QC solutions for oximetry consist of a mixture of dyes simulating the absorbance readings of deoxy-, oxy-, carboxy- and methaemoglobin mixture over a wide range of clinically important concentrations (1). Commercially available QC materials should be handled and prepared for analysis according to manufacturer's recommendations. Newer blood gas analyzers have an on board QC cartridges automated QC capability and/or use "electronic QCs". Auto QCs consist of on board QC materials that are automatically analyzed by the instrument at designated time intervals (regulatory or manufactur- er's). Such QCs may check the test system's analytical components completely or partially, and may be used in combination with traditional external QCs. They monitor temperature, incubation time, correct sample or reagent volume transfer, integrity of the sample (blood clots), and report to the operator failures of acceptable operational limits. In these cases, some analysers may even automatically initiate and document corrective actions. These integrated QCs are consistent and require little or no operational intervention. It is highly recommended to use commercial QC materials for proper monitoring of system performance (1).

Electronic QCs consist of cartridges that verify the electronic components of BGA analyzers. Since electronic QCs cannot verify the analytical performance of the analyzer, additional commercial QC materials should be analyzed concomitantly (1).

\subsection{Proficiency testing for BGA and related measurements}

Laboratories should participate in proficiency testing (PT) programs for BGA and related measurements organized by the Croatian centre for quality assessment in laboratory medicine (CROQALM) of the CSMBLM.

Alternatively, if analytes of interest are not provided by the CROQALM, external independent PT providers are suitable.

A minimum of 3 PT cycles per year is recommended, which is the common frequency of BGA for CROQALM.

The obtained PT results should be reviewed by appropriate personnel and the actions taken in case of unsatisfactory performance. These procedures should be documented (48).

\section{Acknowledgements}

The authors would like to thank to Prof. Ana-Maria Šimundić, PhD and Nora Nikolac, PhD for their valuable comments and suggestions on the manuscript.

\section{Potential conflict of interest}

None declared. 


\section{Appendix}

\section{List of comments received by public discussion of the national recommendations for BGA and answers of Working group for blood gas testing}

\begin{tabular}{lll}
\hline Comment & Authors' response
\end{tabular}

\section{COMMENTATOR 1}

Considering that the use of warm, moist towel at a temperature We thank the commentator for this comment. Section 3.3.2 not higher than $42^{\circ} \mathrm{C}$ is not convenient for routine use, I suggest Arterialisation of the skin puncture site for capillary sampling that you explain in detail arterialisation of capillary sample using describes the procedure of using vasodilatory cream for vasodilatory cream.

I suggest revising the recommendation regarding the frequency of quality control procedures in 24 hours, taking into consideration the price of 3 commercial QC samples daily.

arterialisation of the puncture site.

The section 4.9 of the recommendations states: „Each laboratory should design the internal QC analysis strategy in accordance with good laboratory practice." The frequency of QC analysis depends on the workload, acceptability of quality control results, manufacturer's recommendations and regulatory requirements, which is also clearly stated in recommendations. The analysis of 3 QC samples daily, mentioned by the commentator, is merely an example cited by the CLIA requirements, and should not be considered a national recommendation.

\section{COMMENTATOR 2}

Introduction - some analyzers have also co-oxymeters (built in or connected to the analyzer, like the IL Premier 3000, for example). In case of the IL Premier 3000, the co-oximeter is switched on depending on the analyses requested. When the co-oximeter is turned on, the analyzer measures the $\mathrm{sO}_{2}$, while when it is turned off (in cases when only BGA is requested), the analyzer calculates the $\mathrm{sO} 2$ value. The $\mathrm{sO}_{2}$ results are reported interchangeably.

If this is the case, is it necessary to perform a comparability study? Or, considering that requests for measured $\mathrm{sO}_{2}$ are rare, is advisable to always report the calculated $\mathrm{s}_{2}$ result? competences of laboratory staff; also, there are no bachelors of laboratory diagnostics on the list.
The answer to this comment is included in section 4.9 Quality control for blood gas analysis and related measurements, in the paragraph referring to comparability of BGA results.
Table 1 - it has not been specified which document states

We thank for this comment. Table 1 represents a summary of data from the text, i.e. of the literature that describes competences of individual professions in health care in Croatia regarding blood gas analysis and common practice in Croatian laboratories. We have therefore added in the table title a list of references that refer to stated data.

In order to simplify quotation for different levels of education (high school, undergraduate, graduate and postgraduate level), the working group decided to use only a general term for a particular profession that deals with a specific area (e.g., doctor, nurse).

For this reason, the table does not use the term „bachelor of medical laboratory diagnostics" but only the broader term "laboratory technician".

The goal of this section was to show interdisciplinary areas and professions involved in blood gas analysis.

Sample analysis - the option of including data on patient's body temperature and oxygen supply for the calculation of corrected values of BGA is not described. This is especially important for patients in intensive care units.

Is a sample containing a blood clot never acceptable for BGA, or are there special circumstances that allow the reliable measurement of any BGA parameter?
The section 4.4 Sample analysis of the national recommendations was rephrased in order to answer this comment.

The section 4.3 of the national recommendations states: „Blood gas samples containing air bubbles or visible clots are unacceptable for analysis." Therefore, a sample containing a visible clot is not acceptable for BGA, under any circumstances. 
Quality control - comparability using patient's samples - is a patient single arterial blood sample suitable for comparability studies, especially with respect to $\mathrm{pO}_{2}$ ? Could a serum sample or a lithium heparin plasma sample, aliquoted in multiple stoppered containers (depending on the number of the analysers compared) be used for this purpose instead?
The section 4.9 of the recommendations states that an ongoing comparison should be instituted using the same patient sample, in a short time period, on blood gas analysers located closely. Sample (in)stability should be always considered (especially regarding $\mathrm{pO}_{2}$ ). When selecting a sample for comparability control, the manufacturer instructions on sample type recommended for analysis have to be followed.

Sample storage after analysis - it is not specified if BGA samples should be stored after analysis and how long, for traceability purposes only. The CCMB recommendations are available online. analysis.

\section{COMMENTATOR 3}

„A capillary blood sample can replace an arterial sample only when an arterialisation technique is used, although entirely reliable pO2 measurement can be achieved only when an arterial blood sample is used."

Results of the survey on procedures in acid-base testing in Croatian laboratories show that $46 \%$ of laboratories use capillary sample for blood gas analysis in adults. Further, according to national recommendations of the Croatian Society of Medical Biochemistry and Laboratory Medicine for capillary blood sampling, results of the survey showed that as much as $88 \%$ of laboratories never use arterialisation before capillary sampling. Therefore, I think that considerable attention should be given to arterialisation of capillary sample. As your document has a section entitled Procedure for blood gas sampling, I think that procedure of arterialisation should be explained in more detail, because of situations when there's no possibility to collect arterial blood (geriatric patients, burn etc.). Maybe arterialisation procedure could be more accepted and more present in laboratory practice if a more detailed protocol was described. You quote: "detailed procedure of arterialisation and all steps for capillary sampling are described in the national recommendations of the Croatian Society of Medical Biochemistry and Laboratory Medicine for capillary blood sampling", but unfortunately there are no more details than in your text. It should be clarified what is meant by a towel (whether it would be proper to use a sterile disposable gauze), how to ensure temperature not higher than $42^{\circ} \mathrm{C}$, whether dry warm air should be used, or maybe you can suggest which cream to apply (a cream that contains hyperaemic or vasodilatory agent - which are those substances?). In addition, if a cream is used, how and when disinfection of the puncture site is performed?

The document "Competences for issuing the license for masters of science in medical biochemistry and specialists in medical biochemistry and laboratory medicine" states: "COMPETENCES - in addition to all competences of the master of science in medical biochemistry, a specialist in medical biochemistry and laboratory medicine also gained competences...."

So if I understand correctly, this means that Table 1 declares that wherever there is a competent master of science in medical biochemistry, there should also be a competent specialist in medical biochemistry and laboratory medicine present.
We thank for this comment. Section 3.3.2 Arterialisation of the skin puncture site for capillary sampling describes the procedure of using vasodilatory cream for arterialisation of the puncture site.

We thank for this comment. As you said all competences relating to master of science in medical biochemistry are also related to specialist in medical biochemistry and laboratory medicine. Specialist in medical biochemistry and laboratory medicine has additional competences which are specific for that level of education and they are gained during specialization.

However, to avoid putting too much information in the table, we mention only "Master of science in medical biochemistry" but it is obvious that a specialist in medical biochemistry and laboratory medicine also has competences of master of science in medical biochemistry.

More is explained in the text, and the working group believes that the table should remain unchanged in this segment. 
Section 3.2.2. Anticoagulant the use of liquid heparin is mentioned twice in the same context ("Use of liquid heparin should be avoided because of dilution effect on blood gas and related parameters and ability to bind electrolytes, namely calcium." and then again "Liquid heparin anticoagulation has unfavourable dilution and chemical effect on BGA.")

"QC materials sealed in ampoules contain a gas mixture phase in the headspace and must be equilibrated to the appropriate temperature before opening (if room temperature is significantly different from $25^{\circ} \mathrm{C}$ )." - Besides equilibrating to room temperature, should QC materials, sealed in ampoules, be vigorously mixed before analysis to equilibrate gases in the aqueous phase or should it be emphasized to handle and prepare such samples according to manufacturer's recommendations?
We thank for this comment. The text of the recommendation has been modified according to this comment in section 3.2.2.

We thank for this comment. The recommendations were modified according to this comment in the section 4.9 .

\section{References}

1. Clinical and Laboratory Standards Institute. Blood gas and pH analysis and related measurements; Approved Guideline - Second Edition. CLSI document C46-A2. Clinical and Laboratory Standards Institute Wayne, Pennsylvania, USA, 2009.

2. [Povjerenstvo za stručna pitanja HKMB] Available at http:// www.hkmb.hr/povjerenstva/strucna-pitanja.html. (in Croatian) Accessed September 22nd 2014.

3. Casagranda I. Point-of-care testing in critical care: the clinician's point of view. Clin Chem Lab Med 2010;48:931-4. http://dx.doi.org/10.1515/CCLM.2010.191.

4. Sevdalis $N$, Jacklin $R$, Arora $S$, Vincent CA, Thomson RG. Diagnostic error in a national incident reporting system in the UK. J Eval Clin Pract 2010;16:1276-81. http://dx.doi. org/10.1111/j.1365-2753.2009.01328.x.

5. Dukić L, AM Šimundić. Institutional practices and policies in acid-base testing: a self reported Croatian survey study on behalf of the Croatian society of medical biochemistry and laboratory medicine Working Group for acid-base balance. Biochem Med (Zagreb) 2014;24;281-92. http://dx.doi. org/10.11613/BM.2014.031.

6. Mikulcik P. Rapid Analysis - Blood gases and more; 1st English ed. Siemens Healthcare Diagnostics, München, Germany, 2009. Available at: http://www.healthcare.siemens. com. Accessed November 1st 2014.

7. Clinical and Laboratory Standards Institute. Procedures for the collection of arterial blood specimens; Approved standard - fourth edition. CLSI document GP43-A4 (former H11A4). Clinical and Laboratory Standards Institute, Wayne, Pennsylvania, USA, 2004.

8. WHO Guidelines on drawing blood: Best practices in phlebotomy. Available at: http://www.ncbi.nlm.nih.gov/books/ NBK138650/. Accessed October 26th 2014.

9. Ishii S, Miyajima M, Shishido F, Sakuma K. Hematoma after femoral arterial blood sampling: incidence, imaging features, and course of hematoma. J Comput Assist Tomogr 2012;36:257-60. http://dx.doi.org/10.1097/ RCT.0b013e3182483c55.
10. American Association for Respiratory Care. AARC clinical practice guideline. Sampling for arterial blood gas analysis. Respiratory Care. 1992;8:891-7.

11. Zavorsky GS, Cao J, Mayo NE, Gabbay R, Murias JM. Arterial versus capillary blood gases: A meta-analysis. Respir Physiol Neurobiol 2007;155:268-79. http://dx.doi.org/10.1016/j. resp.2006.07.002.

12. Clinical and Laboratory Standards Institute. Procedures and devices for the collection of diagnostic capillary blood specimens; Approved standard - sixth edition. CLSI document GP42-A6. Clinical and Laboratory Standards Institute, Wayne, Pennsylvania, USA, 2008.

13. McKay RJ Jr. Diagnosis and Treatment: Risks of obtaining samples of venous blood in infants. Pediatrics 1966;38:9068.

14. AARC Clinical Practice Guideline. Capillary blood sampling for neonatal and paediatric patients. Respr Care 2001;46:506-13.

15. Vaquer S, Masip J, Gili G, Gomá G, Oliva JC, Frechette A, et al. Earlobe arterialized capillary blood gas analysis in the intensive care unit: a pilot study. Ann Intensive Care 2014;4:11. http://dx.doi.org/10.1186/2110-5820-4-11.

16. Sauty A, Uldry $C$, Debétaz LF, Leuenberger P, Fitting JW. Differences in $\mathrm{pO} 2$ and $\mathrm{pCO} 2$ between arterial and arterialized earlobe samples. Eur Respir J 1996;9:186-9. http:// dx.doi.org/10.1183/09031936.96.09020186.

17. Meites S, Lin SS, Thompson C. Studies on the quality of specimens obtained by skin puncture of children 1. Tendency to hemolysis, and hemoglobin and tissue fluid as contaminants. Clin Chem 1981;27:875-8.

18. [Katalog znanja i vještina za studij medicine]. Available at: http://www.medri.uniri.hr/dokumenti/assets/katalog/klinika.pdf. (in Croatian) Accessed October 21st 2014.

19. [Standardizirani postupci u zdravstvenoj njezi.] Available at: http://www.hkms.hr/data/1316431523_388_mala_ stand.postupci-kompletno.pdf. (in Croatian) Accessed October 21st 2014. 
20. [Kompetencije medicinskih sestara opće zdravstvene njege.] Available at: http://www.hkms.hr/data/1316431477_292_ mala_kompetencije_18062011_kompletno.pdf. (in Croatian) Accessed October 21st 2014.

21. [Kompetencije za izdavanje odobrenja za samostalan rad magistara medicinske biokemije $i$ specijalista medicinske biokemije i laboratorijske medicine], HKMB, 2013. Available at: http://www.hkmb.hr/povjerenstva/strucna-pitanja.html (in Croatian) Accessed October 21st 2014.

22. [Narodne novine 34/05. Pravilnik o načinu obavljanja medicinsko biokemijske djelatnosti uz bolesnika.] Available at: http://www.propisi.hr/print.php?id=3363. (in Croatian) Accessed October 21st 2014.

23. Nikolac N, Šupak-Smolčić V, Šimundić AM, Ćelap I. Croatian Society of Medical Biochemistry and Laboratory Medicine: national recommendations for venous blood sampling. Biochem Med (Zagreb) 2013;23:242-54. http://dx.doi. org/10.11613/BM.2013.031.

24. Lippi G, Blanchaert $N$, Bonini P, Green S, Kitchen $S$, Palicka $V$, et al. Causes, consequences, detection, and prevention of identification errors in laboratory diagnostics. Clin Chem Lab Med 2009;47:143-53. http://dx.doi.org/10.1515/ CCLM.2009.045.

25. Clinical and Laboratory Standards Institute. Procedures for the collection of diagnostic blood specimens by venipuncture; Approved standard-sixth edition, CLSI document H3-A6, Wayne, Pennsylvania, USA, 2007.

26. [HKMB: Standardi dobre stručne prakse. Sadržaj uputnice.] Available at: http://www.hkmb.hr/povjerenstva/strucna-pitanja.html] (in Croatian). Accessed September 22nd 2014.

27. International Organization for Standardization, ISO15189:2012 Medical laboratories - Requirements for quality and competence. 2012.

28. Baird G. Preanalytical considerations in blood gas analysis. Biochem Med (Zagreb) 2013;23:19-27. http://dx.doi. org/10.11613/BM.2013.005.

29. Burnett RW, Covington AK, Fogh-Andersen N, Külpmann WR, Maas AHJ, Müller-Plathe $O$ et al. Approved IFCC recommendations on whole blood sampling, transport and storage for simultaneous determination of $\mathrm{pH}$, blood gases and electrolytes. Eur J Clin Chem Clin Biochem 1995;33:24753.

30. Crawford A. An audit of the patient's experience of arterial blood testing. Available at: http://www.acutecaretesting. org. Accesed October 14th 2013.

31. Wennecke G, Dal Knudby M, eds. Avoiding preanalytical errors - in capillary blood testing. Radiometer Medical Aps, Brønshøj, Denmark, 2009.

32. Wennecke G, Juel G, eds. Avoiding preanalytical errors - in blood gas testing. Radiometer Medical Aps, Brønshøj, Denmark, 2008.

33. Hedberg P, Majava A, Kiviluoma K, Ohtonen P. Potential preanalytical errors in whole-blood analysis: Effect of syringe sample volume on blood gases, electrolyte and lactate values. Scan J Clin Lab Invest 2009;69:585-91. http://dx.doi. org/10.1080/00365510902878716.

34. Davis MD, Walsh BK, Sittig SE, Restrepo RD. AARC clinical practice guideline: blood gas analysis and hemoxi- metry: 2013. Resp Care 2013;58:1694-703. http://dx.doi. org/10.4187/respcare.02786.

35. Küme T, Şişman AR, Solak A, Tuğlu B, Çinkooğlu B, Çoker $C$. The effects of different syringe volume, needle size and sample volume on blood gas analysis in syringes washed with heparin. Biochem Med (Zagreb) 2012;22:189-201. http:// dx.doi.org/10.11613/BM.2012.022.

36. Lima-Oliveira $G$, Lippi $G$, Salvagno GL, Montagnana $M$, Picheth $G$, Guidi GC. Different manufacturers of syringes: $A$ new source of variability in blood gas, acid-base balance and related laboratory tests? Clin Biochem 2012;45:683-7. http://dx.doi.org/10.1016/j.clinbiochem.2012.03.007.

37. Toffaletti JG. Effect of small air bubbles on changes in blood $\mathrm{pO} 2$ and blood gas parameters: calculated vs. measured effects. Available at: www.acutecaretesting.org Accessed January 19th 2013.

38. Lippi G, Fontana R, Avanzini P, Sandei F, Ippolito L. Influence of spurious hemolysis on blood gas analysis. Clin Chem Lab Med 2013;51:1651-4. http://dx.doi.org/10.1515/cclm-20120802.

39. Salvagno GL, Lippi G, Gelati M, Guidi GC. Hemolysis, lipaemia and icterus in specimens for arterial blood gas analysis. Clin Biochem 2012;45:372-3. http://dx.doi.org/10.1016/j. clinbiochem.2011.12.005.

40. Hess $C E$, Nichols $A B$, Hunt WB, Suratt PM. Pseudohypoxemia secondary to leukemia and thrombocytosis. N Engl J Med 1979;301:361-3. http://dx.doi.org/10.1056/ NEJM197908163010706.

41. Knowles TP, Mullin RA, Hunter JA, Douce FH. Effects of syringe material, sample storage time, and temperature on blood gases and oxygen saturation in arterialized human blood samples. Resp Care 2006;51:732-6.

42. Leniček Krleza J, Dorotić A, Grzunov A, Maradin M. Capillary blood sampling: national recommendations of the Croatian society of medical biochemistry and laboratory medicine. Biochem Med (Zagreb) 2015;25;335-58. http://dx.doi. org/10.11613/BM.2015.034

43. Narayanan S. Preanalytical issues related to blood sample mixing. Available at: http://acutecaretesting.org/en/ articles/preanalytical-issues-related-to-blood-samplemixing. Accessed March 23th 2015.

44. Burtis CA, Ashwood ER, Bruns DE editors. Tietz Textbook of Clinical Chemistry and Molecular Diagnostics - 5th edition. Philadelphia: Elsevier Saunders, 2012.

45. [HKMB. Pohrana i priprava uzoraka, PPSP-ZK 2, izd1.] Available at: http://www.hkmb.hr/povjerenstva/arhiva/ HKMB\%20PPSP\%202.pdf. (in Croatian). Accessed March 26th 2016.

46. Dimeski G, Badrick T, John AS. Ion Selective Electrodes (ISEs) and interferences - a review. Clin Chim Acta 2010;411:30917. http://dx.doi.org/10.1016/j.cca.2009.12.005.

47. Ehrmeyer SS. The importance of quality control (QC) to qu ality blood gas testing. Available at http://acutecaretesting. org/en/articles/the-importance-of-quality-control-qc-toquality-blood-gas-testing. Accessed February 15th 2015.

48. National Association of Testing Authorities. Interpretation of NPAAC Requirements and ISO 15189 Medical Testing Field Application Document. Available at: http: http://www. nata.com.au. Accessed October 7th 2015. 